\title{
Prediction of soil effects on GPR signatures
}

\author{
Jan B. Rhebergen, Henk A. Lensen, René van Wijk ${ }^{a}$, \\ Jan M.H. Hendrickx, Remke van Dam, Brian Borchers ${ }^{b}$ \\ ${ }^{a}$ TNO Physics and Electronics Laboratory, The Hague, The Netherlands \\ ${ }^{b}$ New Mexico Tech, Socorro, New Mexico, USA
}

\begin{abstract}
In previous work we have shown that GPR signatures are affected by soil texture and soil water content. In this contribution we will use a three dimensional electromagnetic model and a hydrological soil model to explore in more detail the relationships between GPR signatures, soil physical conditions and GPR detection performance. First, we will use the HYDRUS2D hydrological model to calculate a soil water content distribution around a land-mine. This model has been verified against measured soil water distributions in previous work. Next, we will use existing pedotransfer functions (e.g. Topp, Peplinski, Dobson, Ulaby) to convert the predicted soil water contents around the land-mines as well as known soil textures and bulk densities into soil parameters relevant to the electromagnetic behaviour of the soil medium. This will enable a mapping between the hydrological model and the electromagnetic GPR model. Using existing and new laboratory and field measurements from the land-mine test facilities at TNO-FEL we will make a first attempt to verify our modelling approach for the prediction of GPR signatures in field soils. Finally a detection algorithm is used to evaluate the GPR detection performance with respect to changing environmental soil conditions.
\end{abstract}

Keywords: Mine detection, soil water distribution, modelling, land mine, anti tank mine, anti personnel mine, test facility, relative permittivity, dielectric constant, ground penetrating radar, GPR, detection algorithm

\section{INTRODUCTION}

Detection performance of mine detection sensors in general is influenced by several environmental factors like, soil texture and structure, soil water content and prevailing and historic weather conditions, as well as like type and depth of the landmine. For a ground penetrating radar (GPR) specifically, the most important parameter that influences GPR operation and performance is the soil water content, since this directly influences the electromagnetic parameters to which a GPR is sensitive namely the permittivity and electrical conductivity of the embedding soil environment.

Through proper modelling using known quantities like bulk soil density and texture, as well as (historic) weather information like precipitation and temperature, the spatial moisture distribution around a buried object like for instance a land-mine can be calculated.

In previous work ${ }^{1}$ we have shown that hydrological modelling of the soil environment around a land-mine closely matches measurements taken during controlled experiments at the TNO-FEL test facilities. ${ }^{2}$

In a follow up step we now couple the hydrological modelling outcome to an electromagnetic model by means of existing pedotransfer functions. In previous work ${ }^{1}$ we have used simple one dimensional GPR reflection time modelling together with Topp's equation ${ }^{3}$ to link model and measurements. In this paper we have chosen to use the equation presented by Dobson, Peplinski and Ulaby. ${ }^{4}$ Alternatively one could use mixing formulas as described by Ari Sihvola. ${ }^{5}$ Instead of the simple one dimensional GPR model we now use a three dimensional electromagnetic finite difference time domain (FDTD) model. ${ }^{6}$ The latter model more realistically predicts GPR behaviour in a soil environment and is also more suitable to linking to the hydrological model. Finally we apply a detection algorithm based on autoregressive modelling ${ }^{7}$ of the mine signature, to determine the effect of the changes in the soil environmental conditions on the GPR detection performance. This AR model extracts eigenmodes from the scattered signal of a mine, and determines the optimum number of poles describing the time series that represents the mine signature. Subsequently the statistical distance of these poles to those of other (measurement) data is determined. Where this distance is minimal we declare a detection.

Send correspondence to: J.B. Rhebergen, TNO-FEL, P.O. Box 96864, NL-2509 JG The Hague, The Netherlands, Rhebergen@fel.tno.nl, phone: +31 703740772 


\section{MODELS}

In this study we use several models, equations and detection algorithms that we will describe here. To elucidate the relationship between the various models we present figure 1. The known quantities are the basic parameters of the environment and

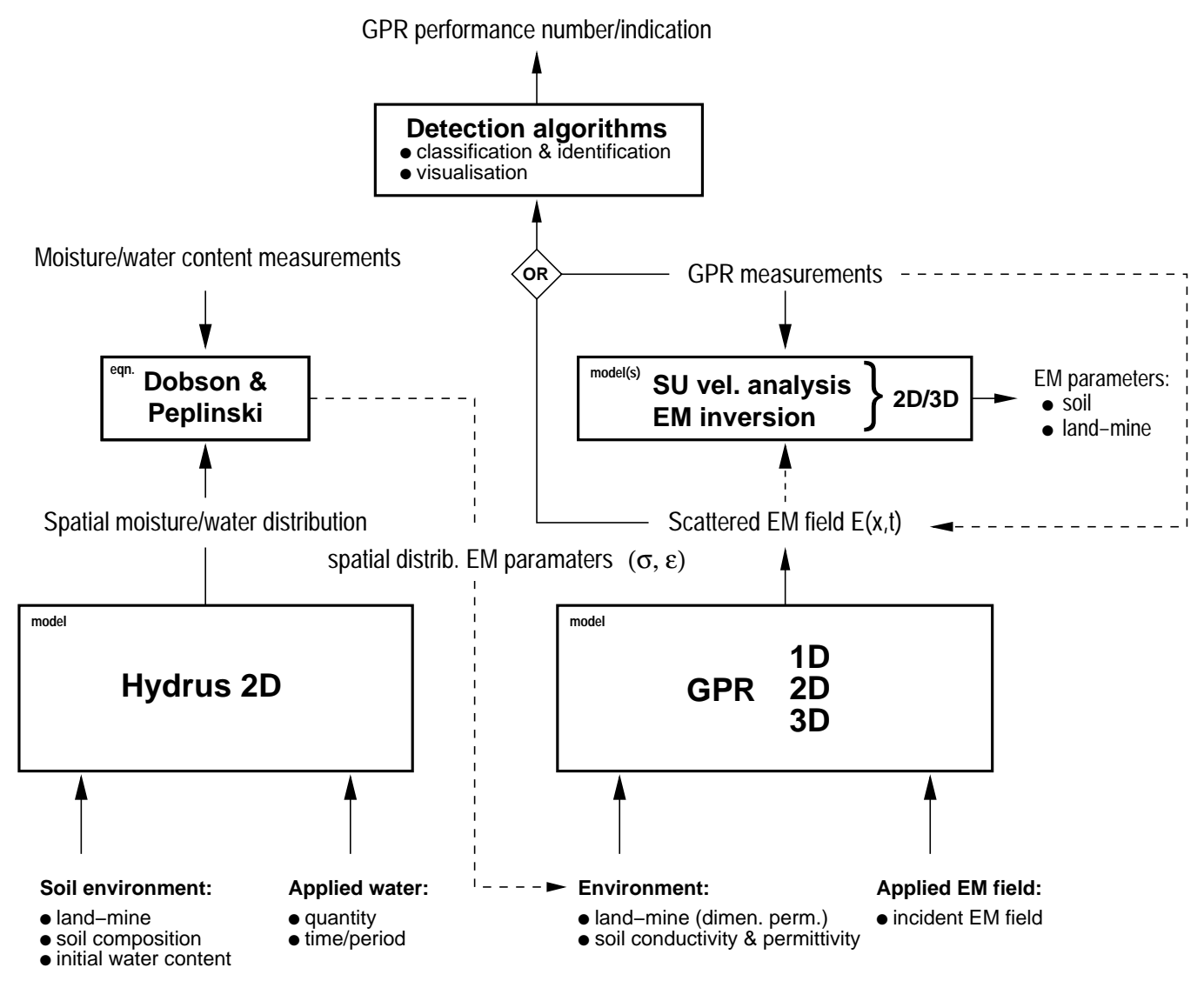

Figure 1. Model relations

the equipment. Input parameters required for the hydrological model would include information such as soil composition, initial water content, applied water, evaporation, etc. For the electromagnetic (EM) GPR model the relative permittivity $\varepsilon_{r}$ and conductivity $\sigma$ of the embedding soil should be known, as well as the incident field applied by the GPR. For both models the location and dimensions of the land-mine also have to be known. In the case of the EM model we also need electromagnetic parameters of the materials the mine is made up of. In our research and experiments we use land-mine simulants containing a compound ${ }^{8}$ which resembles the EM characteristics of real explosives closely.

To evaluate the effect of changes in the environment on the performance of a GPR the whole chain of models is followed starting with the hydrological model. We use HYDRUS 2D in a rotationally symmetric configuration which yields a spatial distribution of the water content around a buried land-mine. Next we link the output of the hydrological model to the electromagnetic model by means of equations proposed by Dobson, Peplinski ${ }^{4}$ and Ulaby. We constructed some Matlab scripts to facilitate easy calculations for a wide range of soil-water contents. The electromagnetic modelling code is a straight forward generally available finite difference time domain (FDTD) code with a choice of different absorbing boundary conditions. ${ }^{6}$ We use the results from the hydrological model after being processed by the Matlab scripts implementing the Dobson, Peplinski and Ulaby equations, to generate the proper embedding environment parameters for the FDTD code. The electromagnetic modelling code subsequently produces data that can be compared to regular GPR measurements obtain from experiments at the TNO-FEL test facility. To determine the effect of a change in soil environment input parameters, we let the different models run and let the effects ripple through the linked models. Just like normal GPR measurements we now apply a detection algorithm to determine if there is an improvement or deterioration in GPR performance. 
Another application of this work is the use of electromagnetic inversion codes. Inversion techniques are notoriously ill posed and often non-unique problems. Some of the problems involved here can be addressed by using the information from the other models advantageously. This however is a topic for another paper and beyond the scope of this one.

\subsection{Hydrological model HYDRUS 2D}

The hydrological modelling as described here enables one to relate known soil characteristics and climatological information to a soil water content distribution around buried objects like land-mines. In this paragraph, we briefly summarise results from earlier work.

Soil water distributions around land-mines have been modelled using the HYDRUS-2D package ${ }^{9}$ of the U.S. Salinity Laboratory at Riverside, California. HYDRUS-2D is a modelling environment for simulating two-dimensional water, heat, and solute movement and root water uptake in variably saturated soil. The flow equations are solved numerically using a Galerkin-type linear finite element scheme.

We have followed earlier work by $\operatorname{Das}^{10}$ et al. and Hendrickx ${ }^{11}$ et al. and used a quasi three-dimensional region exhibiting radial symmetry about the vertical axis. Richards equation was solved to simulate unsaturated water flow in a soil cylinder with a radius of $2 \mathrm{~m}$ and depth of $2 \mathrm{~m}$. The anti tank mine (type NR26) was placed in the centre of the cylinder with its top at a depth of $0.30 \mathrm{~m}$. Observation points were located approximately one centimetre above and one centimetre below the mine about $1 \mathrm{~cm}$ away from its centre. Simulations were conducted for a sand soil similar to that present at the experimental test facility 'Waalsdorp' TNO Physics and Electronics Laboratory in The Netherlands. ${ }^{2}$ Since no hydraulic properties have been measured the hydraulic properties of the Van Genuchten model provided by the HYDRUS-2D model were used. ${ }^{9}$

The initial condition was set as a dry sand soil (4\% volumetric water content) throughout the profile. $80 \mathrm{~mm}$ of water infiltrating in the second hour after start of the simulations was applied as top boundary condition. A no-flux boundary condition was imposed on two sides of the flow domain. The simulations were conducted for a period equivalent to 48 hours infiltration time. It was assumed that no significant evaporation occurred from the soil during this period, which is reasonable for winter conditions in The Netherlands.

Figure 2 presents the changes in soil water content at depths 10, 30, and $45 \mathrm{~cm}$ for the infiltration experiment. The water contents at 30 and $45 \mathrm{~cm}$ depths are those just above and just below the centre of the mine. Although water is applied

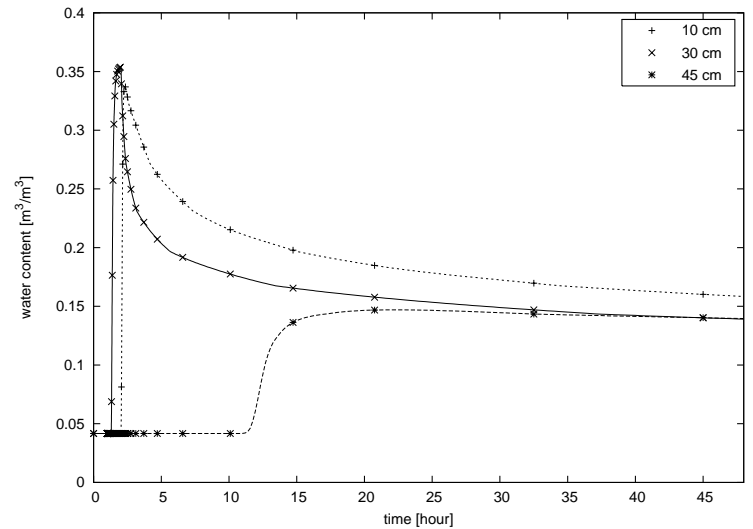

(a) Modelled

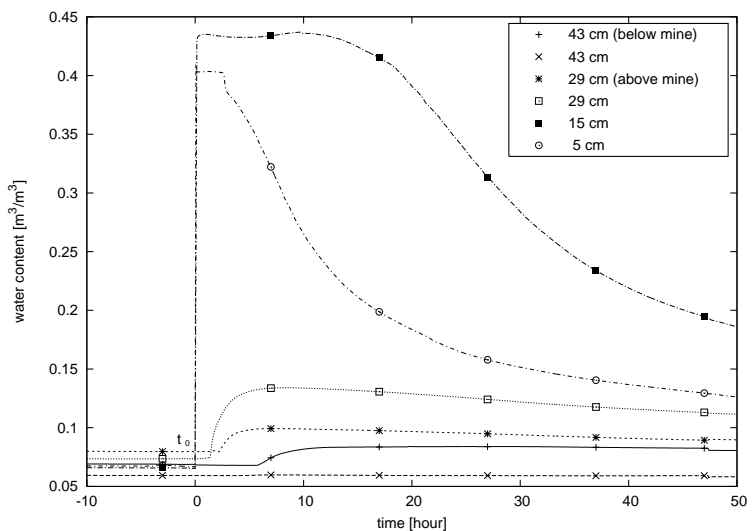

(b) Measured

Figure 2. Soil water content

only during one hour, soil water remains dynamic for the entire 48 hours of the simulation. After an application of $80 \mathrm{~mm}$ the soil water content just above the mine ( $30 \mathrm{~cm}$ depth) increases almost immediately while below the mine ( $45 \mathrm{~cm}$ depth) it takes about 10 hours after termination of water application at the soil surface for the water content to increase. All water 
contents stabilise at around 15 volume percent. This means that a layered soil profile due to water infiltration in a dry soil is a common phenomenon of field soils.

The model outcome was experimentally verified by a corresponding infiltration experiment that was carried out at the TNO Physics and Electronics Laboratory's mine detection test facility 'Waalsdorp'. ${ }^{2}$ Detailed description of this experiment can be found in previous work ${ }^{1}$ (see references).

\subsection{Soil property models}

The dielectric properties of soil depend on a number of factors, including bulk density, the texture of the soil particles (sand, silt, clay), the density of the soil particles, the volumetric water content of the soil, the temperature, and the frequencies of interest. Research has also shown that the dielectric properties of soil depend on the amount of "bound water" which is in close contact with the minerals in the soil. Theoretical and empirical models of the dielectric properties of the different components of the soil have been combined into semi-empirical mixing models which can be used to predict dielectric properties of field soils.

We here summarise the 1995 model of Peplinski, Ulaby and Dobson. This model is a variant of the earlier model of Dobson, Ulaby Hallikainen and El-Rayes. The earlier model was calibrated for frequencies in the range of 1.4 to $18 \mathrm{GHz}$. The model discussed here was calibrated by fitting the model to a set of experimental observations with a variety of soil textures, soil water contents and frequencies from 0.3 to $1.3 \mathrm{GHz}$.

The inputs to this model consist of the volumetric water content $\theta$, the frequency $f$, the fraction of sand particles $S$, the fraction of clay particles $C$, the density of the soil particles $\rho_{S}$ (typically $2.6 \mathrm{~g} / \mathrm{cm}^{3}$ ), and the bulk density of the soil $\rho_{B}$. An empirically determined formula for effective conductivity is,

$$
\sigma_{\text {eff }}=0.0467+0.02204 \rho_{B}-0.4111 S+0.6614 C .
$$

The sand and clay fractions also enter the model through two constants which depend on the soil type but are independent of frequency and soil water content.

$$
\begin{aligned}
\beta^{\prime} & =1.2748-0.519 S-0.152 C \\
\beta^{\prime \prime} & =1.33797-0.603 S-0.166 C
\end{aligned}
$$

The complex dielectric constant (i.e. relative permittivity) for 'free'-water is given by,

$$
\varepsilon_{f w}=\varepsilon_{f w}^{\prime}-\varepsilon_{f w}^{\prime \prime} i,
$$

where the real part is,

$$
\varepsilon_{f w}^{\prime}=\varepsilon_{w_{\infty}}+\frac{\varepsilon_{w_{0}}-\varepsilon_{w_{\infty}}}{1+\left(2 \pi f \tau_{w}\right)^{2}}
$$

and the imaginary part,

$$
\varepsilon_{f w}^{\prime \prime}=\frac{2 \pi f \tau_{w}\left(\varepsilon_{w_{0}}-\varepsilon_{w_{\infty}}\right)}{1+\left(2 \pi f \tau_{w}\right)^{2}}+\frac{\sigma_{\text {eff }}}{2 \pi \varepsilon_{0} f} \frac{\rho_{S}-\rho_{B}}{\rho_{S} \theta} .
$$

In these formulas the $\varepsilon_{0}$ is the permittivity of free space (vacuum), $\varepsilon_{w_{0}}$ is the static dielectric constant (i.e. relative permittivity) of water $\left(=80.1\right.$ at $\left.20^{\circ} \mathrm{C}\right), \varepsilon_{w_{\infty}}$ the high frequency limit of $\varepsilon_{f w}^{\prime}(=4.9)$, and $\tau_{w}$ the relaxation time of water $\left(=9.23 \times 10^{-12} \mathrm{~s}\right.$ at $\left.20^{\circ} \mathrm{C}\right)$. The relative permittivity of soil particles is given by the empirical model,

$$
\varepsilon_{s}=\left(1.01+0.44 \rho_{s}\right)^{2}-0.062
$$

Finally, the real and imaginary part of the relative permittivity for the bulk soil are estimated by,

$$
\varepsilon^{\prime}=1.15\left[1+\frac{\rho_{B}}{\rho_{S}}\left(\varepsilon_{S}^{\alpha}-1\right)+\theta^{\beta^{\prime}} \varepsilon_{f w}^{\prime}{ }^{\alpha}-\theta\right]^{\frac{1}{\alpha}}-0.68
$$

and,

$$
\varepsilon^{\prime \prime}=\left[\theta^{\beta^{\prime \prime}} \varepsilon_{f w}^{\prime \prime}\right]^{\frac{1}{\alpha}}
$$


In these formulas, $\alpha=0.65$ is a constant which has been empirically fitted to the data and the conductivity is given by equation 1.

The texture information of the sand soil present at the TNO-FEL test location, is given in table 1. The clay, silt and sand fractions comprise grain sizes smaller than $2 \mu \mathrm{m}$, between 2 and $63 \mu \mathrm{m}$, and above $63 \mu \mathrm{m}$, respectively. It follows that the sand percentage is $98 \%$. The organic matter content is less than $1 \%$. For the soil particles we assumed a density of $2.6 \mathrm{~g} / \mathrm{cm}^{3}$. The dry bulk density was measured for three samples (at $0 \mathrm{~m}, 0.5 \mathrm{~m}$, and $1.0 \mathrm{~m}$ depth) and averages $1.55 \mathrm{~g} / \mathrm{cm}^{3}$. The gravimetric water content was estimated for the same samples, and was used to calculate the average volumetric water content. The volumetric water content, which is an input parameter in the pedotransfer functions, is $0.083 \mathrm{~cm}^{3} / \mathrm{cm}^{3}$ for the sand soil. Both the dry bulk density and the volumetric water content were measured in the field soil, before excavation and installation in the test lane. We subsequently use the equations that were mentioned above to calculate the complex

Table 1. Grain size fractions obtained after sieving (for material $>63 \mu \mathrm{m}$ ) and using a sedigraph (for material $<63 \mu \mathrm{m}$ ). The analyses were done using samples of the soil before excavation. Tabulated are mean values for the entire soil profile $(0-1.2 \mathrm{~m})$ as installed in the TNO-FEL test-lane.

\begin{tabular}{clc} 
Grain size fractions: & Weight percentage: \\
\hline$>250$ & $\mu \mathrm{m}$ & $34 \%$ \\
$125-250$ & $\mu \mathrm{m}$ & $62 \%$ \\
$63-125$ & $\mu \mathrm{m}$ & $2 \%$ \\
$2-63$ & $\mu \mathrm{m}$ & $1 \%$ \\
$<2$ & $\mu \mathrm{m}$ & $1 \%$
\end{tabular}

permittivity of the actual soil environment that was used in the experiments. The following parameters were used,

Table 2. Soil model input parameters

$$
\begin{aligned}
f & =1 \mathrm{Ghz} \\
\theta & =0.01-0.50 \mathrm{~m}^{3} / \mathrm{m}^{3} \\
\rho_{B} & =1.54 \mathrm{~g} / \mathrm{cm}^{3} \\
\rho_{S} & =2.66 \mathrm{~g} / \mathrm{cm}^{3} \\
\mathrm{~S} & =0.98 \\
\mathrm{C} & =0.01
\end{aligned}
$$

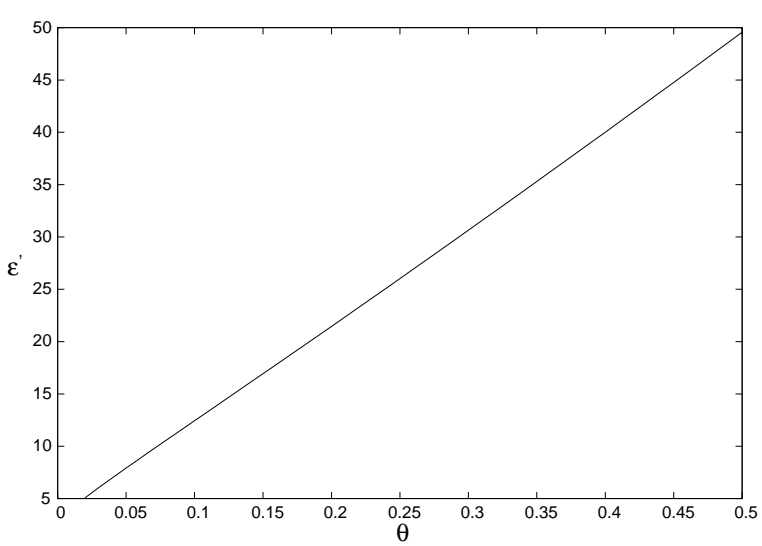

(a) calculated $\varepsilon_{r}^{\prime}$ (real)

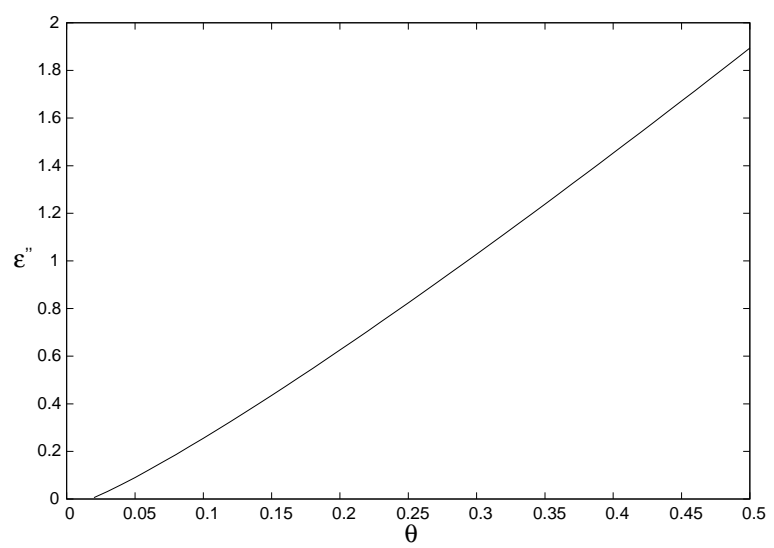

(b) calculated $\varepsilon_{r}^{\prime \prime}$ (imaginary)

Figure 3. Calculated values for the complex relative permittivity $\varepsilon_{r}$ 
At $\theta=0.01$, the model produces an incorrect value. The imaginary part is essentially zero, but the regression model ${ }^{4}$ actually produces a small negative value.This reflects a round off error in the calculations and is there for ignored.

\subsection{Electromagnetic FDTD model}

For the electromagnetic modelling of the GPR environment there are several choices at our disposal. Apart from locally at TNO-FEL developed codes several other options are available. It was decided to use a publicly available FDTD code from Aulbert. ${ }^{6}$ This program, including sources, is placed under the GNU General Public License and free for anyone to use or modify. The mathematical implementation is excellent but creating complicated models is a bit cumbersome.

For the soil environment we used a relative permittivity $\varepsilon_{r}=4$ and for the NR26 mine we used a $\varepsilon_{r}=3{ }^{8}$ Note that we also modelled the air gap and the top structure of the mine (see fig. 4a). Being limited by the rectangular grid we had to approximate the sloping top of the mine with two steps. Figure $4 \mathrm{~b}$ depicts the numerical domain of the FDTD simulation. The mine is placed at a depth of $30 \mathrm{~cm}$ below the surface in the middle of the $150 \times 150 \times 150 \mathrm{~cm}^{3}$ numerical domain. The

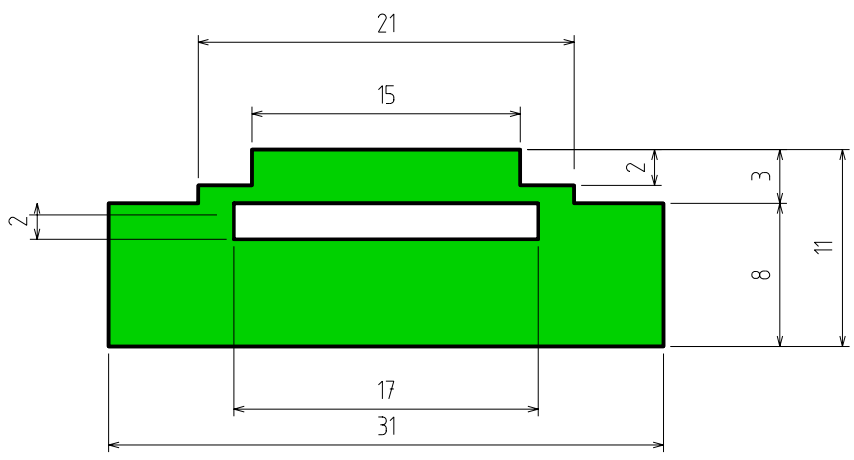

(a) Modelled NR26 AT-mine

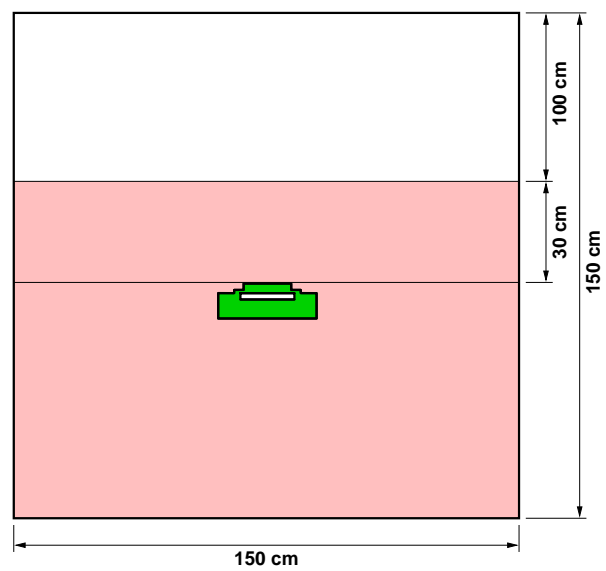

(b) Numerical domain

Figure 4. Modelled NR26 mine plus environment

simulation was carried out with a spatial resolution of $1 \mathrm{~cm}$. Exitation was implemented by means of one and a half periode of sinusoidal cycle stimulus of resp. $100 \mathrm{MHz}$ and $1 \mathrm{Ghz}$. This transmitter is equivalent a dipole generating an $E_{x}$ field. Consequently the receivers (i.e. observation points) were also chosen to record only the $E_{x}$ component. Snapshots of the $\mathrm{XZ}$ plane were taken every 10 steps. The complete simulation was carried out over the equiavalent of $30 \mathrm{~ns}$. The following figures show some results of simulation snapshots. A small conductivity of respectively $\sigma=0.02 \mathrm{~S} / \mathrm{m}$ and $\sigma=0.05 \mathrm{~S} / \mathrm{m}$ was given to the surounding soil medium. For the sake of brevity we only we only show a number of snapshots that demonstrate the refraction of the incident field through the buried land-mine $\left(\sigma_{\text {soil }}=0.05 \mathrm{~S} / \mathrm{m}\right)$.

\subsection{Detection algorithm}

Several GPR mine detection algorithms have been implemented at the TNO Physics and Electronics Laboratory. The one we currently favour is detection by means of autoregressive modelling. ${ }^{7}$ This techniques has also been successfully employed in speech processing. Basically we extract the eigenmodes from the scattered signal of a mine. This will be useful if the models are identifiable. To this end we must specify a unique set of model parameters.

In an autoregressive model of order $R$, a time series $y_{n}$ is modelled as a linear combination of $R$ earlier values in the time series, with the addition of a correction term $x_{n}$ :

$$
y_{n}^{\text {model }}=x_{n}-\sum_{j=1}^{R} a_{j} y_{n-j} .
$$



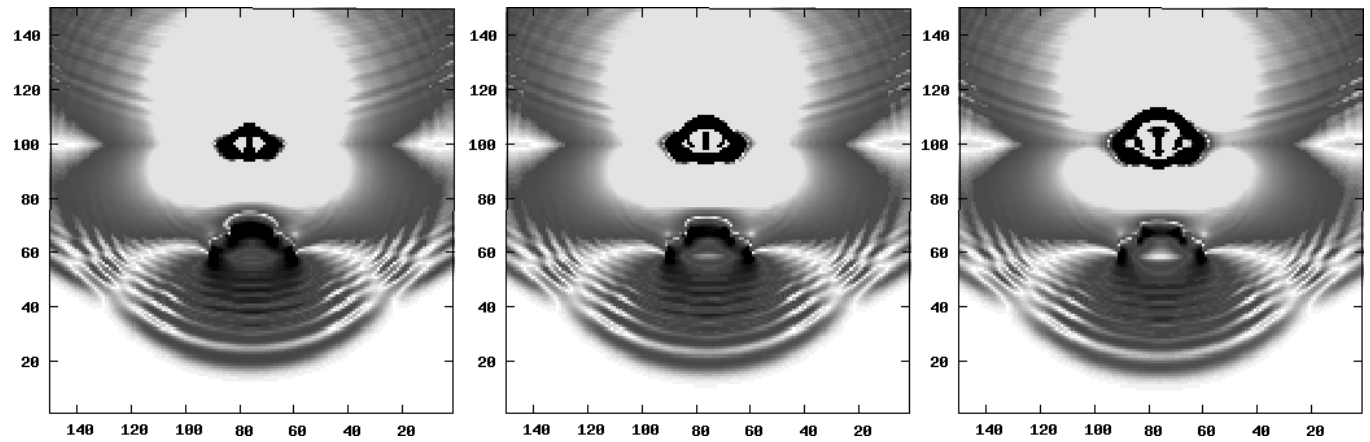

Figure 5. Snapshots of FDTD simulation $(100 \mathrm{MHz})$
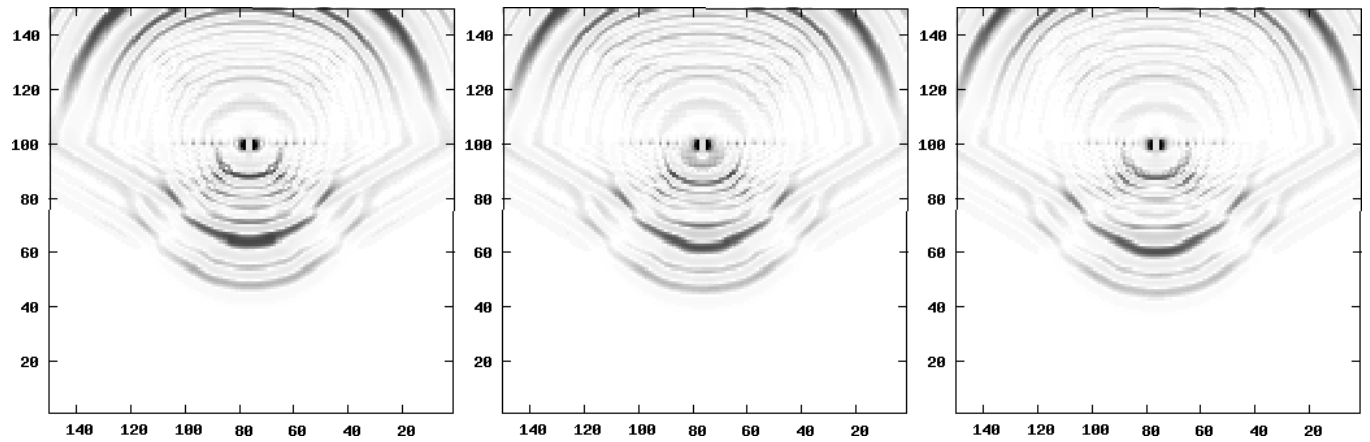

Figure 6. Snapshots of FDTD simulation $(1 \mathrm{GHz})$

The autoregressive coefficients $a_{j}(j=1, \cdots, R)$ are fit by minimising the mean-squared difference between the modelled time series $y_{n}^{\text {model }}$ and the observed time series $y_{n}$.

To render the data analysis using autoregressive models suitable to a wide class of land-mines, we must generalise the estimation of eigenmodes to an arbitrary order of the models. Autoregressive processes of any order can be decomposed into eigenmodes with characteristic oscillation periods. To obtain insight in the reliability of the estimated eigenmodes, confidence intervals for the eigenmodes must be computed from the estimated parameters. In the context of real-time processing, a computational efficient method for estimating the parameters was implemented. To this end, a stepwise least squares algorithm was used. In such an algorithm the model coefficients are computed and evaluation is done by criteria for selection of the model order, which best fits the observed data.

Recognition of an unknown mine is performed using a statistical distance to score the autoregressive model of each mine based upon the given observation sequence, and select the autoregressive model of the mine whose score is highest.

We refer to the theory described by van $\mathrm{Wijk}^{7}$ for the mathematical foundations of this algorithm and only present the results of the application of the algorithm to our data from the infiltration experiment. ${ }^{1}$

To begin with we build an autoregressive (AR) model of the signal coming from a know mine location. Suppression of any undesirable and unwanted signal artefacts will lead to a better more representative model of the mine. Removal of the direct coupling signal for instance, would be a good starting point. We therefore select only a limited part of the land-mine related GPR trace. As input parameters to the AR model we also need to specify the minimal and maximal order of the all-pole model. In figure 7 we depict part of the GPR trace which has the mine signature in it, as well as the corresponding spectrum, impulse response and location of the poles describing the AR model. Next we determine the statistical distance of the mine AR model with respect to the other data in the GPR track and plot this in the same picture depicted in figure 8. Where this quantity is minimal we declare a detection. As can be clearly seen from the figure the statistical distance between the AR model and the data is a minimum at the mine locations. The detection algorithm even is able to pick out the mine signature from between the buried TDR sensors.

Next we present data taken at two instances. The first set was taken right after the start of the infiltration experiment. The second one was the final measurement taken, roughly two weeks after the start of the infiltration experiment. Looking 

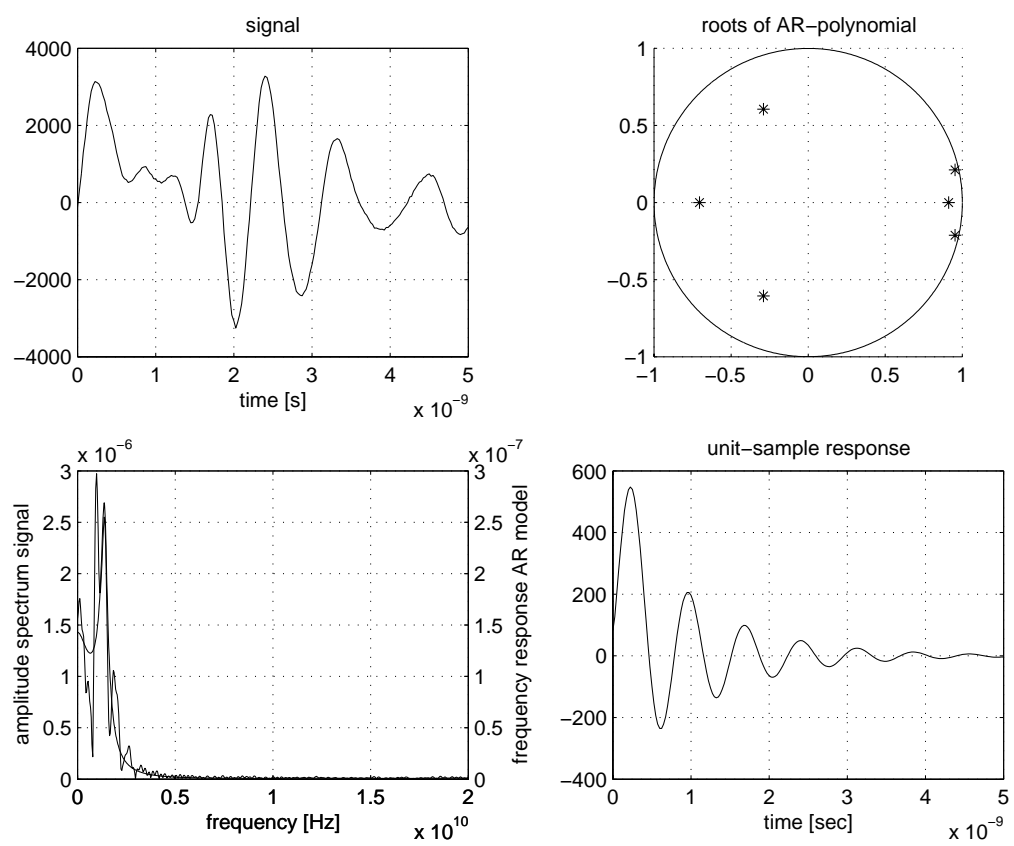

Figure 7. Mine related part of GPR signal together with poles describing the corresponding AR model (amplitudes are specified in arbitrary units).
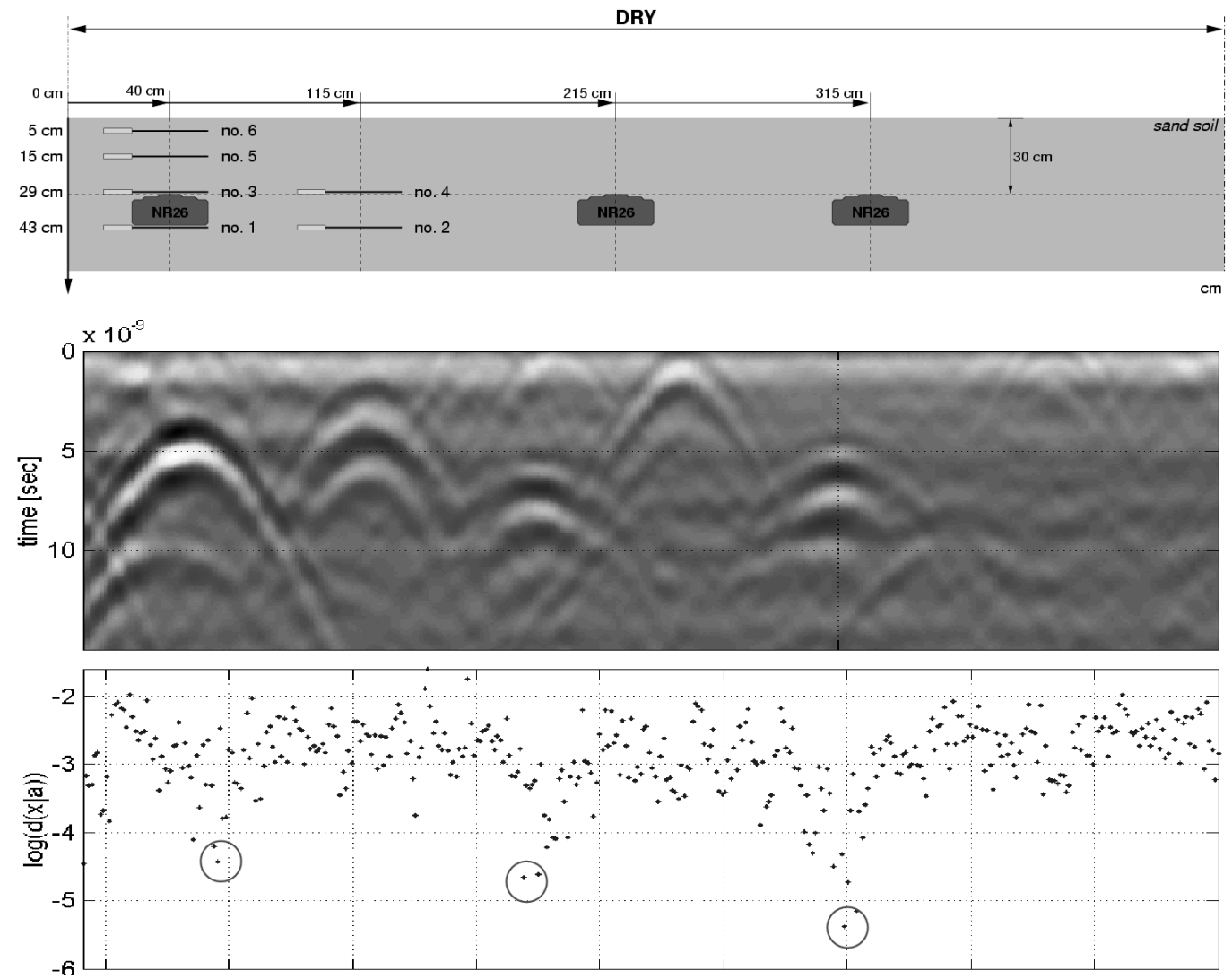

Figure 8. Actual mine position(s) together with raw GPR data and statistical distance between AR model and data. 

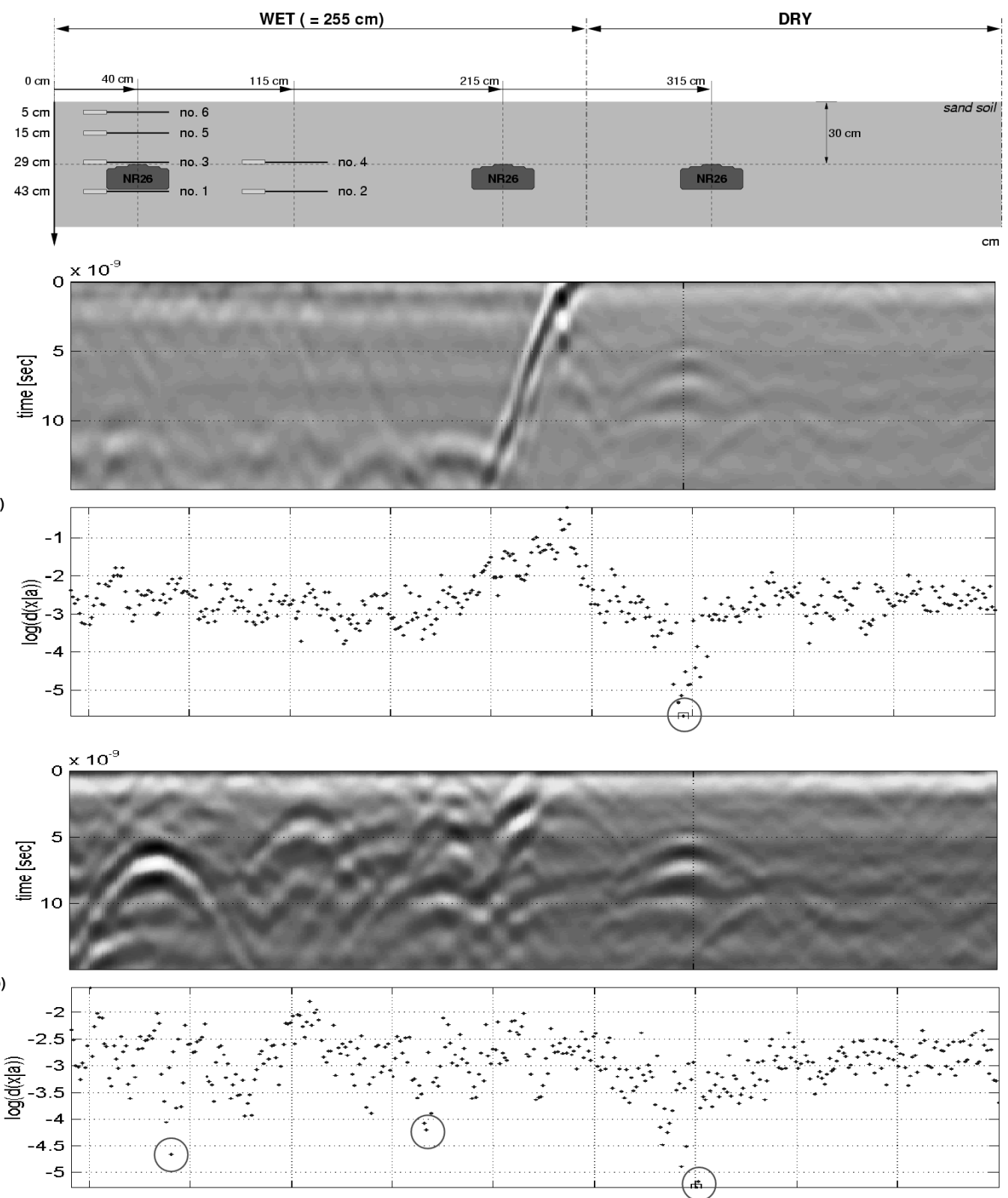

Figure 9. Actual mine position(s) together with raw GPR data and statistical distance between AR model and data. First taken right after the start of infiltration, second at the end of the experiment. 
at figure 9a) we see that the EM wave propagation speed of the sand declines as a result of the increased water content. The result is that the part of the GPR signal that contains information of the AT mine has shifted down further in time i.e. partly out of the data acquisition window. Too little information is now present in the data to be able to build a reliable AR model and detect the mine. In figure 9b) we see the situation two weeks after the start of the infiltration experiment. The wave propagation speeds have increased again, resulting in better data with more information pertaining to the mine. Looking at figure 2b) we see a moisture level (water content) of close to $15 \%$. With the naked eye an untrained observer will have trouble to identify the location of the (wet) mine from this data. However judging from the graph with the statistical distances the detection algorithm seems to pick up the mine signature again with a minimum at the respective mine locations.

\section{CONCLUSIONS}

We have proposed a scheme of linked models and algorithms that can be used to evaluate the detection performance of a ground penetrating radar under varying environmental soil conditions. Next we have illustrated the use of these models for the problem of mine detection using data from experiments with land-mine simulants. We have also demonstrated how these models can be linked together to form a processing chain. Finally we have shown how soil water content influences the detection performance of a GPR by means of a detection algorithm. From experiments and modelling it is clear that (increasing) water content adversely affects detection performance. It is demonstrated that, with knowledge of the soil environment and some other parameters, one can predict a soil moisture distribution around a land-mine, which in turn can be used as input to an electromagnetic model, that predicts the GPR response under those environmental soil circumstances.

The work presented here has been carried out for one soil type and one type of land-mine (and GPR). Future work will focus on expanding the range of soil types and land-mines. The link between the different models is currently based on parameter mapping but can, and should be, implemented more tightly, more accurate and over a bigger spatial domain. Different candidate detection algorithms should also be evaluated. Further validation by means of extended numerical and physical experiments is envisaged. To be of practical use, a future version of this collection of linked models has to be calibrated and trained on real world scenarios.

\section{ACKNOWLEDGMENTS}

This material is based upon work supported by the U. S. Army Research Office under Prime Grant No. DAAD19-02-1-0274 and by the Royal Netherlands Army Countermine research programme. The authors would like to thank Dr. Russell S. Harmon, Senior Program Manager at the Army Research Office, for his valuable advice and support.

\section{REFERENCES}

1. J. B. Rhebergen, H. A. Lensen, S. P. B. W., J. M. H. Hendrickx, and G. Rodríguez Marín, "Soil water distribution around land mines and the effect on dielectric contrast," in Detection and Remediation Technologies for Mines and Mine-like Targets VII, Proceedings of the SPIE, SPIE, Orlando, April 2002.

2. J. B. Rhebergen, "Mine-detection test facilities at tno-fel site 'waalsdorp': Some examples of results obtained with an array based gpr system," Subsurface Sensing Technologies and Applications 3, pp. pp. 369-385, October 2002.

3. G. C. Topp, J. L. David, and A. P. Annan, "Electromagnetic determination of soil water content: Measurements in coaxial transmission lines," Water Resources Research 16, pp. 574-582, June 1980.

4. N. P. Peplinski, F. T. Ulaby, and M. C. Dobson, "Dielectric properties of soils in the 0.3 - 1.3 ghz range," IEEE Transactions on Geosience and Remote Sensing 33, pp. 803-807, 1995.

5. A. Shivola, Electromagnetic mixing formulas and applications, Electromagnetic Waves, IEE, London, 1999.

6. C. Aulbert, "Effizient absorbierende randbedingingen in numerischen bodenradarsimulationen mittels "generalised perfectly matched layers"," Master's thesis, TU Braunschweig, Institut für Geophysik und Meteorologie, Juli 2000.

7. C. V. van Wijk, "Signal modelling using autoregressive models," Signal Processing 82, pp. 1829-1844, December 2002.

8. Dow Corning Europe, Brussels, Product Information for Silastic 3110 RTV Silicone Rubber, 1981. Data Sheet number 61-143-01. 
9. J. Šimůnek, M. Šejna, and M. T. van Genuchten, HYDRUS-2D/MESHGEN-2D code for simulating water flow and solute transport in two-dimensional variably saturated media. International Ground Water Modeling Center, Colorado School of Mines, CO 80401, USA., gwmc - tps 53c, ver. 2.0 ed.

10. B. S. Das, J. M. H. Hendrickx, and B. Borchers, "Modeling transient water distributions around landmines in bare soils.," Soil Science 166, pp. 163-173, 2001.

11. J. M. H. Hendrickx, B. S. Das, and B. Borchers, "Modeling distributions of water and dielectric constants around landmines in homogeneous soils.," in Detection and Remediation Technologies for Mines and Minelike Targets IV, Proceedings of the SPIE 3710, pp. 728-738, 1999. 